\title{
Understanding Symptoms in RYR1-Related Myopathies: A Mixed-Methods Analysis Based on Participants' Experience
}

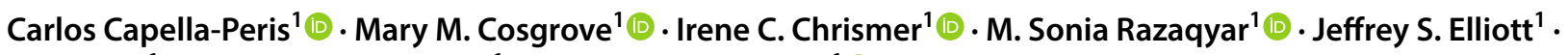 \\ Anna Kuo ${ }^{1} \cdot$ Magalie Emile-Backer $^{1} \cdot$ Katherine G. Meilleur $^{1} \mathbb{B}$
}

Published online: 23 April 2020

(c) Springer Nature Switzerland AG 2020

\begin{abstract}
Background In rare diseases such as ryanodine receptor 1-related myopathies (RYRI-RM), health-related quality of life (HRQoL) measures are critically important so clinicians and researchers can better understand what symptoms are most important to participants, with the ultimate goal of finding tangible solutions for them.

Objectives The main objective of this study was to characterize symptoms in individuals with RYRl-RM to inform future research. A secondary objective of this study was to analyze positive and negative sentiments regarding symptoms and treatment effects post $\mathrm{N}$-acetylcysteine (NAC) administration in individuals with RYRI-RM.

Methods The study used a mixed-methods design applying methodological triangulation. Qualitative data were collected via semi-structured interviews at three visits to characterize symptoms in individuals with RYRI-RM and to analyze treatment effects. Qualitative data were then transformed into quantitative results to measure the frequency with which each symptom was mentioned by participants.

Results A total of 12 symptoms were identified as areas of interest to participants with RYRI-RM, highlighting fatigue and weakness as key symptoms. Data transformation categorized more than 1000 citations, reporting a greater number of positive comments for postintervention interviews than for baseline and preintervention visits and that NAC group participants stated more positive comments regarding treatment effect than did the placebo group.

Conclusions We present a comprehensive characterization of symptoms in RYRl-RM and how those symptoms influence HRQoL. Furthermore, the introduction of mixed methods may be a valuable way to better understand patient-centered data in rare diseases to support affected individuals in coping with their symptoms.
\end{abstract}

Electronic supplementary material The online version of this article (https://doi.org/10.1007/s40271-020-00418-7) contains supplementary material, which is available to authorized users.

Carlos Capella-Peris

carlos.capellaperis@mail.nih.gov; capellac@uji.es

1 Neuromuscular Symptoms Unit, Tissue Injury Branch, National Institute of Nursing Research, National Institutes of Health, Building 60, Room 254, Bethesda, MD 20892, USA

\section{Key Points for Decision Makers}

Symptoms of individuals with ryanodine receptor 1-related myopathies (RYRI-RM), a rare disease, were systematically characterized from a patient perspective to inform and support affected individuals, family members, and healthcare providers.

A specific research design was applied to better understand patient-centered data to promote its application in future studies of RYRI-RM.

This approach meets US FDA guidance on the inclusion of affected individuals' feedback in developing patientreported outcome measures and clinical trials for drug development. 


\section{Introduction}

In recent years, a burgeoning transition has taken place from traditional clinical research methods that are centered on study goals to the inclusion of input from affected individuals in the research process. This change emphasizes a direct approach to measuring how affected individuals feel and function and highlights the value of patient-centered data, not only for developing patient-reported outcome (PRO) measures [1] but also for drug development [2].

Patient-centered data and research are becoming increasingly recognized and are informing regulatory decisions, health policies, research design, and research cost analyses [3]. They also provide important insights into affected individuals' experience as it relates to their functional outcomes, responses to disease symptoms or treatment side effects, and/or an overall assessment of quality of life (QoL). Health-related QoL (HRQoL) looks at these facets through the lens of disease and medical care.

Where treatments have not yet been discovered, HRQoL measures are particularly important so clinicians and researchers can better understand what disease effects are most important to participants, with the ultimate goal of finding tangible solutions for them [4, 5]. Additionally, in smaller populations such as rare diseases, PRO trial endpoints become more critical in targeting the most significant symptoms [6]. Therefore, selecting appropriate PRO measures offers a unique added value not only to understanding the impact of medical conditions and treatments from the affected individuals' perspective but also to ensure any impact of a trial intervention would be comprehensively assessed and meaningful, supporting trial interpretations. This requires the development of specific instruments to address the effects of rare diseases in their limited population rather than using existing generic HRQoL measures. Results from our research will enhance the development of these tools for neuromuscular disorders.

Overall, PROs enrich data to improve future clinical trial methods, study design, participant selection, and biomedical outcomes [7, 8]. Furthermore, PROs help enable positive research experiences that enhance future recruitment, another challenge in rare disease [9-11]. However, quantitative PRO data have known limitations in neuromuscular diseases, such as the "disability paradox" (i.e., affected individuals with neuromuscular disease selfreport a high QoL on HRQoL scales, whereas their family, caregivers, or external observers report them as having a low QoL) [12-15]. It is therefore important to add qualitative analyses to studies to better understand this issue.

Mixed-methods analysis, which combines quantitative and qualitative approaches to provide a more comprehensive understanding of data, has been proposed as the best practice to address patient-centered data in rare diseases since this methodology allows the use of data from small sample sizes [16, 17]. Recent guidance by the US FDA have also provided industry recommendations for ensuring patient perspectives are captured in clinical research [18]. Therefore, we explored the application of mixed methods to a study of individuals affected with ryanodine receptor 1-related myopathies (RYRI-RM).

$R Y R I-\mathrm{RM}$ are caused by pathogenic variants in the RYRI gene, which is highly intolerant to change and encodes the major calcium channel in skeletal muscle [19-21]. RYRIRM comprise the most common form of congenital myopathy, with a pediatric incidence of $>1: 90,000$ in the USA [21-23]. Common RYRI-RM clinical manifestations include proximal/axial muscle weakness, delayed motor milestones, impaired mobility, pain, and fatigue [23-29]. Although affected individuals report fatigue as one of the more pervasive symptoms [25], these symptoms vary greatly in severity. Currently there is no FDA-approved treatment for RYRl-RM [20]. For this reason, it was necessary to perform additional research to characterize symptoms, especially regarding treatment effects and symptom alleviation.

The application of mixed methods in RYRI-RM research to incorporate participants' perspectives on their symptoms may inform future PRO measures and maximize our understanding of RYRI symptoms. Both qualitative (exploratory primary endpoint) and quantitative (secondary endpoint) PRO methods were employed during a combined natural history study and clinical trial of N-acetylcysteine (NAC) (NCT02362425) to facilitate an understanding of the affected individuals' point of view regarding their symptoms and to analyze treatment effects.

\section{Materials and Methods}

\subsection{Standard Protocol Approvals, Registrations, and Patient Consents}

The RYR1 NAC clinical trial consisted of two components: a prospective natural history assessment and a parallelgroup, randomized, double-blind, placebo-controlled trial. The trial incorporated a comparison of quantitative and qualitative PRO data to identify symptoms and subjective improvements post NAC treatment (Harmonic ${ }^{\circledR}$, BioAdvantex Pharma Inc., Toronto, ON, Canada). Given the potential therapeutic efficacy of NAC for RYRI-RM [30-32], NAC or placebo were administered orally for 6 months (adult dose $2700 \mathrm{mg} /$ day; pediatric dose $30 \mathrm{mg} / \mathrm{kg} /$ day). At the conclusion of the trial, no difference in the primary outcome measure for oxidative stress was observed. Results from the 6-minute walk test were $24 \mathrm{~m}$ longer on average in 
the NAC arm, but this did not reach statistical significance [20]. The study was conducted at the National Institutes of Health (NIH) Clinical Center, Bethesda, MD, USA, between 2015 and 2017. This was the first clinical trial conducted in this patient population. The clinical trial is registered in the US National Library of Medicine (NCT02362425). All procedures were approved by the NIH combined neuroscience institutional review board, and an independent monitoring committee was established to oversee trial safety. All participants and parents of participants age $<18$ years provided written informed consent, according to the Declaration of Helsinki, before enrollment. Assent was also obtained for those aged $<18$ years. The total study duration was 18 months and consisted of a 6-month natural history assessment, followed by a 6-month intervention phase, and a follow-up phone call at 18 months. Participants attended three study visits at the NIH: baseline, preintervention, and postintervention. Randomization was performed at the end of the second visit. For more information regarding the clinical trial, see Todd et al. [20].

\subsection{Study Design}

An exploratory sequential mixed-methods design with methodological triangulation was employed, QUAL $\rightarrow$ (quan) $[33,34]$. The mixed-methods approach was recently recommended by the FDA to incorporate patients' perspectives in clinical research [18]. Qualitative data were collected via semistructured interviews at each visit to characterize symptoms in individuals with RYRI-RM and to analyze treatment effects. Thus, individual and collective viewpoints were addressed. This design has previously been implemented in HRQoL and neuromuscular disorders (NMD) research [35-37] as well as in symptoms research [38]. Qualitative data were used to assess the importance and depth of comments from the participants. Then data transformation was applied to obtain quantitative results. In this standard procedure of mixed-methods research, investigators take the qualitative themes or codes and count them to form quantitative measures [34, 39]. Therefore, data transformation was used to measure the frequency with which each area of interest was mentioned by participants.

\subsection{Study Goals}

The primary goal of this study was to characterize symptoms in individuals with $R Y R I$-RM to inform future research. As a secondary goal, this study aimed to analyze positive and negative sentiments regarding symptoms and treatment effects post NAC administration in individuals with RYRI-RM.

\subsection{Research Questions and Hypotheses}

The research questions and hypotheses were as follows:

$\mathrm{Q}_{1}$ : What are the major symptoms in individuals affected with RYRI-RM?

$\mathrm{Q}_{2}$ : How do these symptoms impact individuals affected with RYRI-RM pre- and post-NAC administration?

We hypothesized that data transformation would identify a greater number of positive comments in individuals with RYRI-RM for postintervention interviews compared with baseline and preintervention interviews and that NAC group participants would mention more positive comments than placebo group participants regarding treatment effect.

\subsection{Participants}

Overall, 150 individuals were screened for participation in this study, 53 of whom were eligible and enrolled (Fig. 1). Of these, 47 participants completed at least one interview that we were able to include in the qualitative analysis. Therefore, a criterion-i purposeful sampling approach was used [40]. Although 27 postintervention interviews were performed, the two specific questions to assess treatment effect were not asked in one of those interviews. Therefore, the number of participants available in which to assess treatment effect was 26, with 13 in each arm of the study. Demographic information is presented in Table 1 .

\subsection{Procedures}

\subsubsection{Qualitative Analysis}

A total of 107 semistructured interviews were conducted using open-ended questions [see the electronic supplementary material (ESM)]. Two postintervention questions were added at the final visit. Interviews were conducted between March 2015 and November 2017 (baseline), September 2015 and January 2017 (preintervention), and March 2016 and June 2017 (postintervention). Interviews had an average duration of $13 \mathrm{~min}$. All interviews were conducted at the NIH by the principal investigator, a research nurse, or a research fellow. All interviews were recorded and transcribed verbatim to conduct content analysis [41], applying a multiphase approach, open coding, and axial coding [42]. Content analysis usually involves converting qualitative data into a quantitative form through frequency counts [39]. This was implemented in our research design, and this procedure is described in Sect. 2.6.2. Three researchers analyzed an initial set of five interviews to identify common areas of interest (domains/subdomains) and to assess agreement. After confirming agreement, the remaining interviews were distributed among the researchers for analysis. Three additional meetings were conducted to discuss the inclusion of 


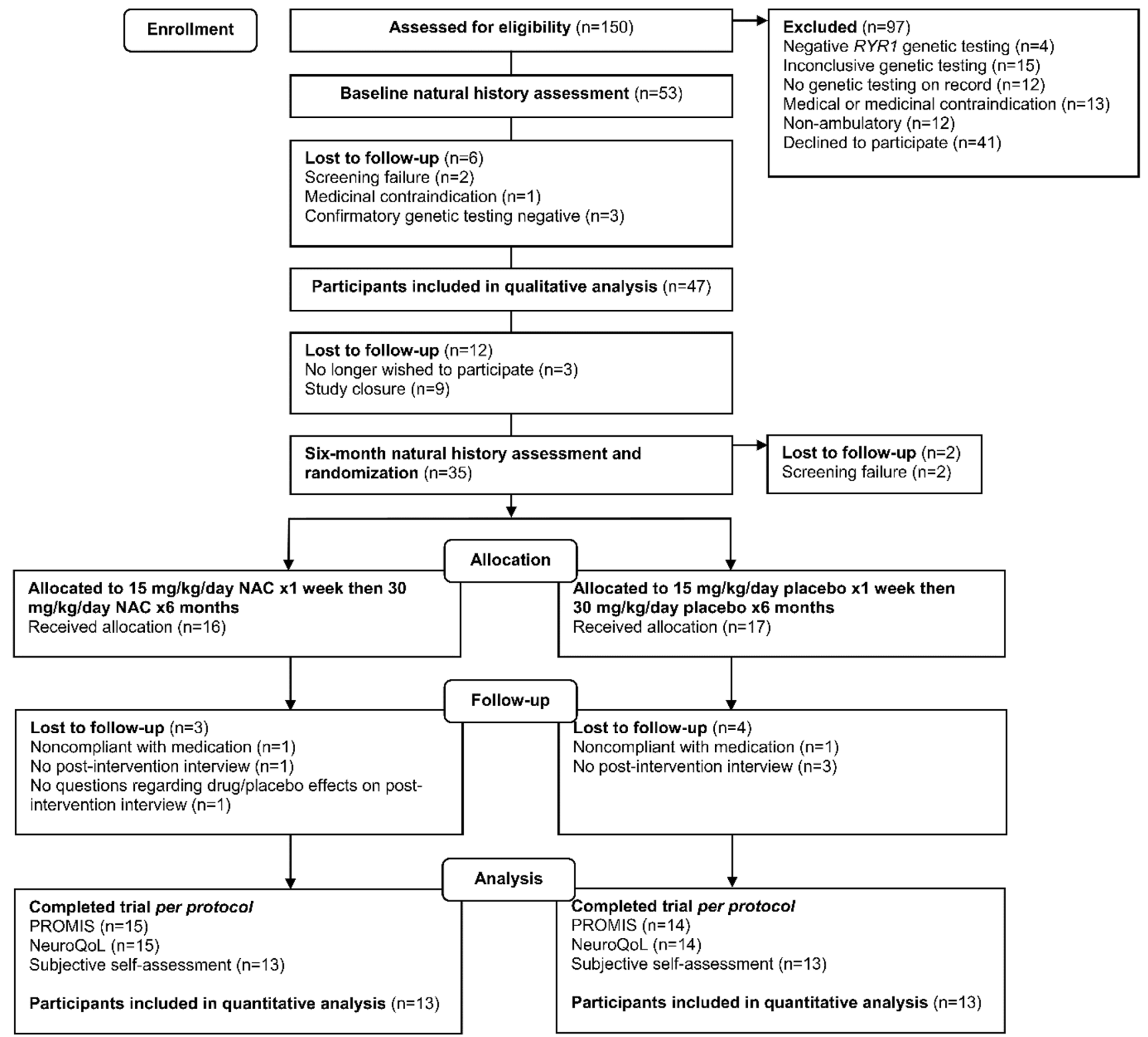

Fig. 1 Consort diagram of study flow. $N A C N$-acetylcysteine, RYRl ryanodine receptor 1

new domains/subdomains until saturation was achieved [43]. Differences in coding were resolved through consensus and tighter definitions of subdomains [44]. Likewise, interpretation and selection of quotes for publication were also discussed to ensure agreement among researchers.

\subsubsection{Data Transformation and Sentiment Analyses}

To perform data transformation, the number of times each domain/subdomain was mentioned in semistructured interviews was counted [36]. Those counts were used to calculate the average and the percentage of citations for each domain/ subdomain. When necessary, percentage scores were normalized to compare domains/subdomains. This procedure was conducted globally (i.e., analyzing all data), by domain (i.e., considering every identified domain independently), by visit (i.e., evaluating data for each visit separately), by treatment (i.e., analyzing treatment effect comments for NAC and placebo groups independently), and by participant (i.e., assessing the records provided for all participants individually). A word frequency analysis was also conducted to identify the most cited words from semistructured interviews [45].

To conduct sentiment analyses, all comments related to the identified domains/subdomains, were labeled as either "positive" or "negative." In this process, reports of "no change" regarding treatment effect were categorized as negative sentiments. Very few comments were considered 
Table 1 Participant demographic information

\begin{tabular}{lllll}
\hline Variables & Overall participants & Qualitative analysis & NAC group & Placebo group \\
\hline$N$ & 53 & 47 & 13 & 13 \\
Cohort $\%$ & 100 & 89 & 25 & 25 \\
Sex & & & & \\
Female & $29(55)$ & $27(57)$ & $8(62)$ & $7(54)$ \\
Male & $24(45)$ & $20(43)$ & $5(38)$ & $6(46)$ \\
Age, years & & & & \\
Total & $29.8 \pm 17.6$ & $29.6 \pm 17.1$ & $32.8 \pm 16.3$ & $23.8 \pm 17.6$ \\
Adults $(>18)$ & $35(66)$ & $31(66)$ & $9(69)$ & $6(46)$ \\
& $39.9 \pm 12.7$ & $39.4 \pm 12.4$ & $42.4 \pm 7.3$ & $39.7 \pm 13.2$ \\
Pediatric $(<18)$ & $18(34)$ & $16(34)$ & $4(31)$ & $7(54)$ \\
& $10.2 \pm 2.7$ & $10.5 \pm 2.8$ & $11.0 \pm 2.9$ & $10.1 \pm 2.0$ \\
Ethnicity & & & & \\
White & $47(89)$ & $42(89)$ & $12(92)$ & $13(100)$ \\
Black & $6(11)$ & $5(11)$ & $1(8)$ & $0(0)$ \\
Location & & & & \\
Northeast & $10(19)$ & $10(21)$ & $1(8)$ & $3(23)$ \\
Southeast & $9(17)$ & $9(19)$ & $5(39)$ & $2(15)$ \\
Midwest & $10(19)$ & $5(11)$ & $2(15)$ & $0(0)$ \\
Southwest & $4(7)$ & $4(9)$ & $2(15)$ & $1(8)$ \\
West & $10(19)$ & $10(21)$ & $1(8)$ & $2(15)$ \\
Abroad & $10(19)$ & $9(19)$ & $5(39)$ \\
\hline Da & & & &
\end{tabular}

Data are presented as $N(\%)$ or mean \pm standard deviation unless otherwise indicated $N A C N$-acetylcysteine neutral (i.e., no positive nor negative sentiment) or dual (i.e., positive and negative sentiments at the same time). For this reason, numbers from sentiment analysis slightly differ from the original counts. To ensure the same approach when attributing sentiments to participants' comments, one researcher conducted this task. After completing sentiment analysis, results were reviewed to confirm agreement among researchers.

Nvivo version 12.5 software (QSR International Pty Ltd., Doncaster, VIC, Australia) was used to perform qualitative research, data transformation, and sentiment analyses.

\section{Results}

\subsection{Qualitative Analysis}

Two domains were identified after conducting data analysis: symptoms and postintervention. Twelve subdomains comprised the symptoms domain: fatigue, fine motor, heat intolerance, mobility, muscle spasms, numbness, pain, respiratory difficulties, rhabdomyolysis (comments related to the destruction of striated muscle cells or elevated levels of creatine kinase not directly linked to any other symptom), scoliosis, weakness, and compounding illness-conditions (impacts of other issues that were not caused by the RYRI mutation(s), for example, symptoms that worsened their QoL, such as a cold, cancer, etc.). Postintervention, the identified domain that assessed treatment effect, included two subdomains: treatment benefits and side effects. Table 2 highlights the most representative quotes for each of these domains/subdomains, selected by their importance and depth (i.e., the best described and the most detailed ones, respectively), to exemplify participants' experiences/opinions. As stated, these quotes were selected through consensus to ensure agreement among researchers.

\subsection{Data Transformation and Sentiment Analyses}

This section counts the number of comments associated with every analyzed domain/subdomain from qualitative analysis. In general, more than 1000 citations were categorized and submitted to sentiment analyses. Figure 2 displays the results for the symptoms domain, Table 3 provides the results for analyses by visit, Fig. 3 displays the results for the postintervention domain with treatment analysis, and Fig. 4 presents the results for the word frequency analysis through a word cloud image. This word cloud highlighted the presence of several groups of terms such as "sometimes, always, usually, morning, summer"; "different, trying, difficult, harder, affected"; 
Table 2 Transcripts from the most representative domains/subdomains

Symptoms $>$ Fatigue: participants reported extreme levels of fatigue, greater than what is typically defined as tiredness and impacting them all day. The most commonly reported ways to minimize this effect were reducing physical activity, increasing breaks to rest and recover, using external support tools, and asking other people for help. Fatigue not only impacted participants' physical performance but also restricted social interactions and required psychological adaptations to cope with this effect from the disease. The importance of this symptom highlighted the value of addressing it to reduce limitations and improve HRQoL. Additionally, the impact of fatigue was a top target when assessing treatment effects, reemphasizing the significance of this symptom

"Just being drained by the end of the day. Since I work full time, it's hard to get everything done I need to do at home. Thankfully, he helps me do a lot of the things around the home ... A lot of mornings, even when I wake up, I always have to have eight hours sleep or I just can't function, but I wake up tired ..."<C32VA_CCP $>$ Ref 1-2 [Dual sentiments (positive and negative)]

Symptoms > Weakness: participants also highlighted difficulty in completing several common tasks because of a lack of strength. In addition to reducing their range of movements, this highlighted a need for external support to perform some actions. As before, this physical effect had social and psychological impacts, not only for the participants but also for their family, friends, coworkers, etc. Weakness was closely related to participants' fatigue as well as mobility, reinforcing its identification as one of the most important symptoms for individuals with $R Y R 1$-RM. Weakness was also a top target for treatment assessment

"Not as much as the strength, I would say, because ... my muscles get tired first before I get out of breath. That comes with, like, going up the stairs. Like, when I play sports or if I do exercise, like go to the gym, and usually, I can go further, because I'm just, my muscles are getting tired and getting sore rather than me being out of breath and have to catch my breath" $<$ C26VA_ICC $>$ Ref 2 [Negative sentiment]

Symptoms > Pain: similar to previous symptoms, pain was reported by participants with RYRI-RM as a persistent effect and revealed the impact and difficulty of dealing with symptoms of pain on a regular basis. Pain was described as myalgia, affecting different muscles throughout the body and impairing motor function in daily life activities. This symptom affected not only physical performance but also mood and mental wellbeing

"It's been a little more difficult because it seems like I'm having a little more weakness and a little more pain, which keeps me from being able to do a little bit more than what I could do before ... My legs hurt really bad at movies, where my feet don't touch the floor ... then if it's, to go shopping like we used to, I can't walk" < C17VB_MMC > Ref 1-2 [Negative sentiments]

Postintervention > Treatment benefits: several participants experienced positive effects after using NAC. The most important benefits were focused on fatigue and weakness. Also, some individuals affected by RYRI-RM reported reduced pain. Positive impacts were not limited to physical effects, providing social and psychological benefits as well. Despite the multiple benefits reported, several participants also described no changes in response to drug treatment. Those comments were categorized as negative sentiments

"Before January, I was taking different things_CoQ10 Ubiquinol, and that had seemed to work short-term, but this new thing that we're on worked a lot better ... Considerably better ... now I could probably recover quicker" <C20VC_CCP > Ref 1-2 [Positive sentiments/NAC] "Since I have been on the medicine, the pain seems to have been lessened and more tolerable, and the weakness seems to be somewhat better. But it still exists, but it seems noticeably ... since I've been on the medicine, I do notice that my recovery has been quicker than normal" $<$ C17VC_MMC $>$ Ref 1-3 [Positive sentiments/NAC]

"Not at all, I think I was on the placebo. Like, I genuinely think that I didn't notice a single difference ... I think I was expecting to have more endurance or something, so like going up the stairs with my friends, but I still felt exactly as weak as before. Still just as tired from my normal life ... Yeah, it didn't do anything" $<$ C45VC_ICC $>$ Ref 1-4 [Negative sentiments/placebo]

Postintervention > Side effects: the only reported observation that was possibly related to the drug (i.e., feeling extreme fatigue), because the others were in the placebo group, was described in conjunction with potential benefits, reporting higher energy and more strength in the same comment. This minimized the impact of this finding. Additionally, the remaining two side effects reported by participants were assessed as not serious (i.e., acid reflux and headaches)

"I did have two really extreme points in the last 6 months where I had extreme fatigue and I don't know if it's just because it was the weather change or if it was the drug or what, and then I've had a shift where I've had less fatigue for a couple weeks, too." $<\mathrm{C} 01 \mathrm{VC} \_\mathrm{CCP}>\mathrm{Ref} 1$ [Dual sentiment (positive and negative)/NAC]

"From the medicine I had? Headaches" $<$ C24VC_ICC $>$ Ref 1 [Negative sentiment/placebo]

"Some acid reflux" < C34VC_MMC > Ref 1 [Negative sentiment/placebo]

Researchers' interpretations of participants' interviews are labeled with domain and subdomain (e.g., Symptoms $>$ Fatigue). To represent the voice of individuals affected with RYRl-RM, transcripts of participants' comments are also displayed for each case. A reference code noting the deidentified case number, visit, researcher initials, Nvivo transcript reference number, and sentiment assessment follows each quote. For postintervention interviews, drug assignment is also included at the end of the reference code. Pseudonyms were used to protect patient identity

$N A C N$-acetylcysteine, $R Y R 1$ ryanodine receptor 1-related myopathies

and "physical, activity, working, playing, sports". Those words described in detail when, how, and which activities were more limited for participants because of their symptoms.

Examples of results for analysis by participant are available in Figs. e-1 and e-2 in the ESM.

\section{Discussion}

According to our sentiment analyses, more than $80 \%$ of the comments made by participants while talking about their symptoms were related to negative experiences. This shows that the impact of the disease on participants regarding their symptoms was mainly negative, clearly reducing 
Fig. 2 Data transformation results for symptoms domain $(N=107)$. Total count of mentions (whole bar) with green reflecting positive comments and $r e d$ reflecting negative comments. A+indicates positive comments average per person, $\mathrm{D}+$ indicates overall positive comments percentage for this domain, A - indicates negative comments average per person, D - indicates negative comments percentage for this domain, SD+indicates positive comments percentage for each subdomain, and SD - indicates negative comments percentage for each subdomain

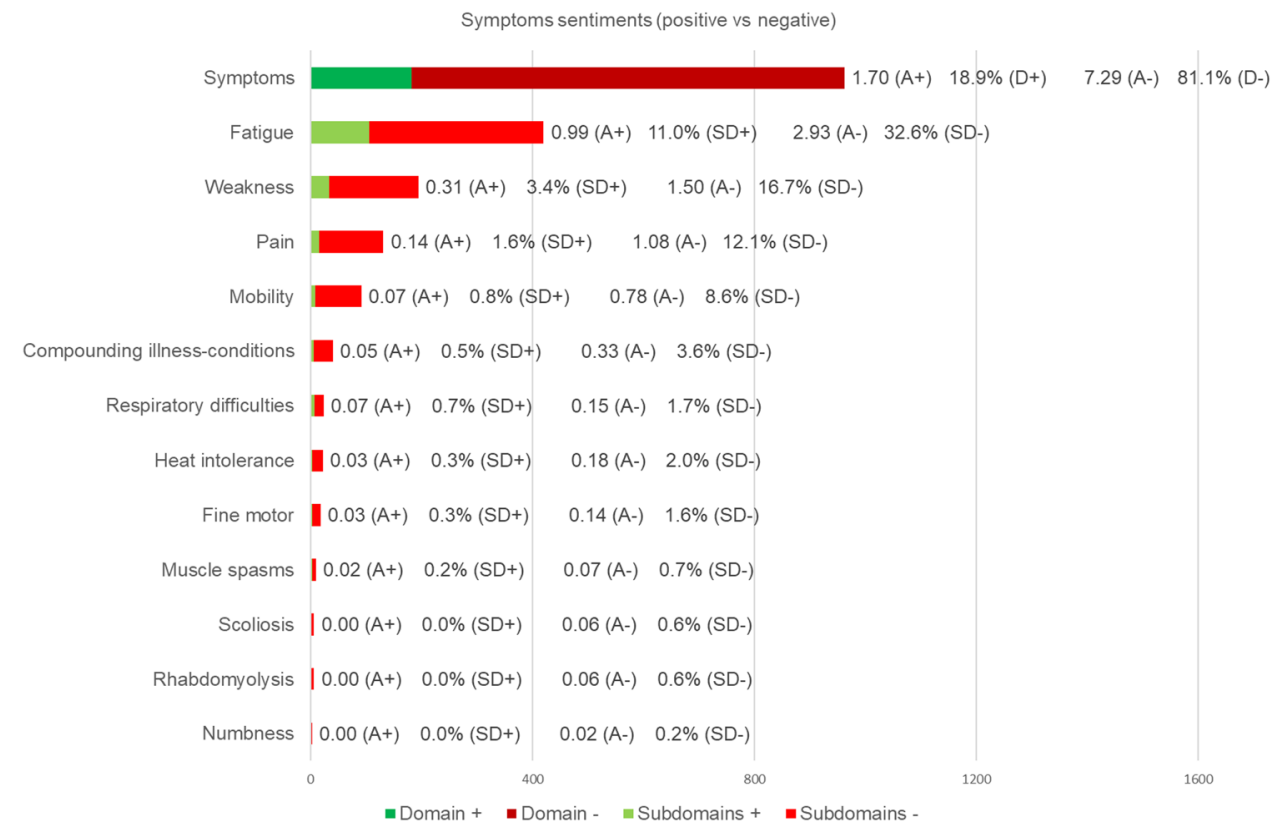

their HRQoL $[46,47]$. However, when considering only the postintervention domain, there was a shift in this trend, displaying $62.1 \%$ of positive comments. This trend was more noticeable in the NAC group $(39.0 \%$ positive vs. $14.9 \%$ negative) than in the placebo group (23.1\% positive vs. $23.0 \%$ negative). The analysis by visit reinforced these results, showing higher positive and lower negative percentages of comments for postintervention interviews compared with baseline and preintervention. Additionally, the analysis by participant and treatment on postintervention domain, revealed that 7 of 13 participants from the NAC group registered more positive than negative comments, of which six reported only one or no positive comments in baseline and preintervention interviews. Regarding the placebo group, only 4 of 13 participants displayed more positive than negative comments for postintervention domain, suggesting little positive effect on this group. Those positive reports maybe due to the placebo effect, which was recently studied in NMD [48, 49]. Together, qualitative and data transformation results suggested a positive trend regarding the effect of NAC in this context. However, as with the 6-minute walk test, this did not reach statistical significance [20]. We should note that participant responses may be biased by positivity and/or social desirability because a study was being done in their rare disease and they felt supported. However, it is important to emphasize that positive comments tripled in number at the postintervention visit compared with the baseline and preintervention visits.

Based on our qualitative analysis, the most important symptom for individuals with RYRl-RM was fatigue, which is in agreement with documented clinical manifestations of this disease $[23,25,50]$. Data transformation revealed that fatigue was the most cited symptom, representing more than $40 \%$ of the total count, which highlighted its importance and agrees with participant reports. The analysis by visit strengthened these findings, revealing consistent results compared with the rest of the data. Specifically, fatigue was the most cited symptom in all visits and displayed similar percentages of positive (approaching to 7\%) and negative (approximately 35\%) statements for baseline and preintervention interviews. On the other hand, on postintervention visit, positive comments tripled in number, whereas negative comments decreased below $30 \%$. Finally, the word frequency analysis exposed the value of terms such as "fatigue/d, stamina, endurance, active, energy, breaks, exhausted, tiredness," reemphasizing the importance of fatigue as a symptom for individuals with RYRI-RM.

Regarding weakness, qualitative analysis revealed this symptom was of secondary importance to participants, which was consistent with previous RYRI-RM research $[23,25,27,29,50-54]$. Although weakness was mentioned half as many times as fatigue, data transformation yielded approximately 200 quotes suggesting that this too was of high importance to participants. The analysis by visit showed a balanced percentage of negative experiences for all visits (around 16.5\%). However, positive statements increased from baseline and preintervention compared with postintervention interviews (from $1.5 \%$ and $0.8 \%$ to $8 \%$ ). Also, weakness registered in $3.4 \%$ of $18.9 \%$ of all positive comments, suggesting that NAC may have also improved strength in participants. Additionally, the word cloud revealed the importance of several words such as "muscle, pushing, strength, weakness, stairs, support, 


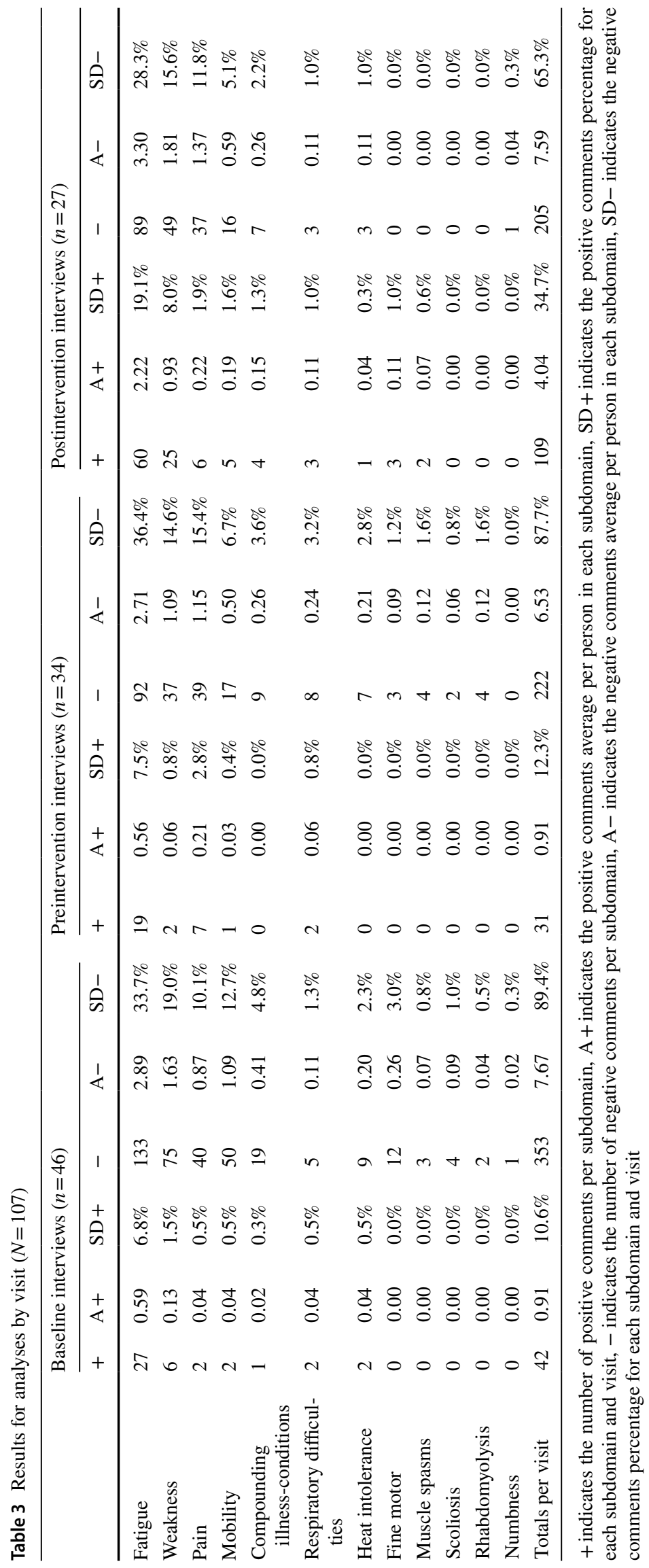




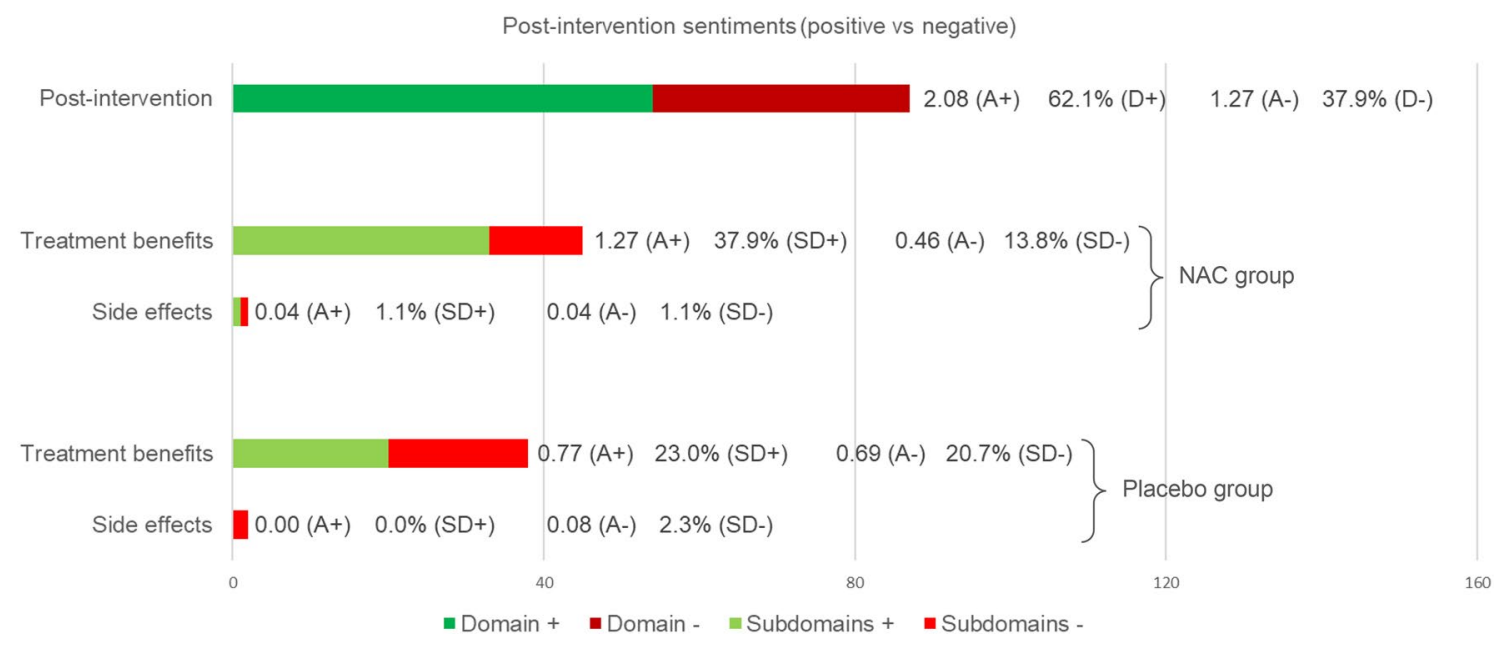

Fig. 3 Data transformation results for postintervention domain with treatment analysis $(N=26)$. Total count of mentions (whole bar) with green reflecting positive comments and red reflecting negative comments, A + indicates positive comments average per person, $\mathrm{D}+$ indicates overall positive comments percentage for this domain,
A - indicates negative comments average per person, D - indicates overall negative comments percentage for this domain, $\mathrm{SD}+$ indicates positive comments percentage for each subdomain and group, and SD - indicates negative comments percentage for each subdomain and group. $N A C N$-acetylcysteine

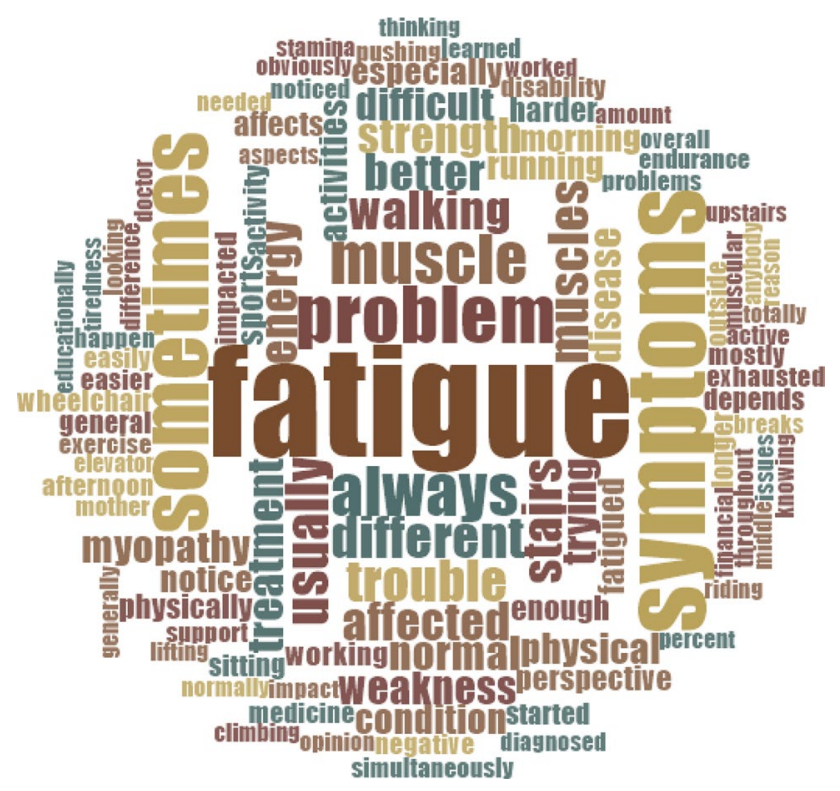

Fig. 4 Word cloud summarizing word frequency analysis $(N=107)$. Larger font sizes indicate a higher frequency of mentions, smaller font sizes a lower frequency

problem, trouble," highlighting the significance of weakness for participants.

Based on qualitative analysis, pain and mobility were on a third level of importance to patients, after other symptoms. They were closely linked to fatigue and weakness as antecedents. Both symptoms produced limitations on motor function and impacted additional areas (i.e., social, psychological, etc.). As before, there is precedence for pain and impaired mobility in affected individuals with RYRl-RM [23-27]. Although pain was cited more times than mobility in the data transformation analysis (131 vs. 91), mobility was also described with great detail in qualitative interviews. The analysis by visit displayed a weak trend of more positive and fewer negative comments in postintervention interviews for mobility. No clear pattern was discernable for pain. This finding supported the idea that NAC had little direct effect on pain and mobility, which is consistent with the fact that these are not indications for NAC therapy [55]. Based on qualitative data, we considered that positive comments regarding pain and mobility in postintervention interviews may be due to improvements in fatigue and weakness. Finally, the word frequency analysis displayed several terms such as "lifting, sitting, climbing walking, running, upstairs, elevator, and wheelchair" that stressed the value of addressing impaired mobility for this population.

The remaining symptoms, fine motor, heat intolerance, muscle spasms, numbness, respiratory difficulties, rhabdomyolysis, scoliosis, and compounding illness-conditions, captured minimal attention in qualitative analysis. They were described as hand dexterity problems, malignant hyperthermia incidents, nonvoluntary muscular contractions, insensitivity feelings, breathing insufficiency, elevated levels of creatine kinase, abnormal curvatures of the spine, and additional limitations, all of which were previously reported in RYRI-RM research [23, 25, 27, 29, 51-54, 56, 57]. Compared with fatigue (420), weakness (194), pain (131), and mobility (91), the remining symptoms were clearly less cited: compounding illness-conditions (40), respiratory difficulties (23), heat intolerance 
(22), fine motor (18), muscle spasms (9), scoliosis (6), rhabdomyolysis (6), and numbness (2). To put this in perspective, all positive and negative citations regarding these symptoms represented just $13 \%$ of mentions for the whole study, suggesting very little impact regarding those symptoms. In the analysis by visit, respiratory difficulties, fine motor, muscle spasms, and compounding illnessconditions displayed a trend of more positive and fewer negative quotes for postintervention interviews. However, the number of comments was very low. Altogether, these findings suggest that these symptoms were linked to individual cases in very few participants. Whilst prior studies stressed the importance of rhabdomyolysis in RYRIRM [24, 56, 57], our data transformation and qualitative results yielded little impact in this symptom. However, it is probable that rhabdomyolysis involvement was also reported by participants within the weakness symptom. Also, rhabdomyolysis may be a more important symptom in the absence of having myopathy, and having myopathy was an inclusion criterion in this research.

Regarding the treatment effect assessment, our qualitative analysis describes that any treatment benefits from NAC were directly linked to reducing fatigue and increasing strength. In addition, some indirect improvements were also accounted for with pain and mobility. On the other hand, only three side effects were identified throughout the entire study.

Additionally, the analysis by visit displayed a higher number of quotes for baseline interviews. However, considering the ratio of participants' comments for each visit, postintervention interviews provided more comments by participant, which may be due to participants' positive expectations regarding the clinical trial [58]. The analysis by participant displayed consistent results compared with global analysis (i.e., analyzing all data), showing similar trends of results for most of the participants.

The main limitation in this study was the small sample size. Additionally, the context in which questions were asked may have shaped participants' responses. Also, because affected individuals report fatigue as one of the more pervasive symptoms, and preclinical data suggested that NAC may impact fatigue, several open-ended questions requested information regarding this symptom specifically. This may have increased the number of mentions for fatigue. In addition, data transformation may have contributed to a loss in depth of data, reducing qualitative data to a binary or frequency. Another limitation may be that all enrolled participants were ambulatory and thus were not representative across the $R Y R I-\mathrm{RM}$ population.

\section{Conclusion}

Our qualitative analysis provides relevant information regarding the major symptoms in individuals with RYRIRM and how those symptoms influence their HRQoL. Data transformation analysis reported a greater number of positive comments in postintervention interviews than in baseline and preintervention visits and that NAC group participants contributed more positive comments regarding treatment effect than did the placebo group. Our findings, especially the identification of fatigue and weakness as key symptoms, the positive effect trend on those symptoms due to NAC treatment, and the description of participants' experiences regarding their symptoms, will inform future studies in this rare disease. In addition, given the FDA's guidance and prioritization on the inclusion of feedback from affected individuals in clinical trials, PRO measures, and drug development [18], the proposed approach is an excellent design for patient involvement in a clinical trial.

Acknowledgements The authors thank all study participants for their sacrifices and commitment to participating in this study. We also appreciate the support provided during the study by Ms. Karez Hawkins, Ms. Monique O. Shelton, and Dr. Joshua J. Todd (Neuromuscular Symptoms Unit, National Institute of Nursing Research, NIH). We would like to thank Dr. Joan K. Austin (School of Nursing, Indiana UniversityPurdue University Indianapolis) for reviewing the manuscript. We acknowledge the RYR1 Foundation for assistance with recruitment for this study.

Author Contributions Conceptualization, funding acquisition, resources, supervision: KGM. Methodology: CC-P and KGM. Formal analysis and investigation: CC-P, MMC, ICC, MSR, JSE, AK, ME-B, and KGM. Original draft preparation: CC-P, MMC, and ME-B. Review and editing: CC-P, MMC, ICC, MSR, JSE, AK, ME-B, and KGM.

Data Availability Statement The datasets generated during and/or analyzed during the current study are available from the corresponding author upon request.

\section{Compliance with Ethical Standards}

Funding This work was supported by the Intramural Programs of the National Institute of Nursing Research, National Institute of Neurological Disorders and Stroke, the NIH Clinical Center, and Bench to Bedside Award [10-2013/Office of Rare Disease/NINR].

Conflict of Interest CC-P, MMC, ICC, MSR, JSE, AK, ME-B, and KGM have no conflicts of interest that are directly relevant to the content of this article.

\section{References}

1. U.S. Food and Drug Administration. Guidance for industry patient-reported outcome measures: use in medical product development to support labeling claims. Rockville; 2009. 
2. U.S. Food and Drug Administration. Patient-focused drug development: collecting comprehensive and representative input. Rockville; 2018.

3. Mercieca-Bebber R, King MT, Calvert MJ, Stockler MR, Friedlander M. The importance of patient-reported outcomes in clinical trials and strategies for future optimization. Patient Relat Outcome Meas. 2018;9:353-67.

4. Landfeldt E, Edström J, Jimenez-Moreno C, van Engelen BGM, Kirschner J, Lochmüller H. Health-related quality of life in patients with adult-onset myotonic dystrophy type 1: a systematic review. Patient-Patient-Centered Outcomes Res. 2019;12:365-73.

5. Simon A, Pompilus F, Querbes W, Wei A, Strzok S, Penz C, et al. Patient perspective on acute intermittent porphyria with frequent attacks: a disease with intermittent and chronic manifestations. Patient-Patient-Centered Outcomes Res. 2018;11:527-37.

6. Logviss K, Krievins D, Purvina S. Characteristics of clinical trials in rare vs. common diseases: a register-based Latvian study. Rosenkranz G, editor. PLoS One. 2018;13:e0194494.

7. Calvert M, Thwaites R, Kyte D, Devlin N. Putting patientreported outcomes on the 'big data road map'. J R Soc Med. 2015;108:299-303.

8. Deshpande P, Sudeepthi Bl, Rajan S, Abdul Nazir C. Patientreported outcomes: a new era in clinical research. Perspect Clin Res. 2011;2:137-44.

9. Slade A, Isa F, Kyte D, Pankhurst T, Kerecuk L, Ferguson J, et al. Patient reported outcome measures in rare diseases: a narrative review. Orphanet J Rare Dis. 2018;13:61.

10. Benjamin K, Vernon MK, Patrick DL, Perfetto E, Nestler-Parr S, Burke L. Patient-reported outcome and observer-reported outcome assessment in rare disease clinical trials: an ISPOR COA emerging good practices task force report. Value Heal. 2017;20:838-55.

11. International Rare Diseases Research Consortium (IRDiRC). Patient-centered outcome measures initiatives in the field of rare diseases (internet). 2016. https://www.irdirc.org/wp-content/uploa ds/2017/12/PCOM_Post-Workshop_Report_Final.pdf

12. Albrecht GL, Devlieger PJ. The disability paradox: high quality of life against all odds. Soc Sci Med. 1999;48:977-88.

13. de Oliveira CM, de Araújo APQC. Self-reported quality of life has no correlation with functional status in children and adolescents with spinal muscular atrophy. Eur J Paediatr Neurol. 2011;15:36-9.

14. Landfeldt E, Lindgren P, Bell CF, Guglieri M, Straub V, Lochmüller $\mathrm{H}$, et al. Health-related quality of life in patients with Duchenne muscular dystrophy: a multinational, cross-sectional study. Dev Med Child Neurol. 2016;58:508-15.

15. Peric S, Vujnic M, Dobricic V, Marjanovic A, Basta I, Novakovic I, et al. Five-year study of quality of life in myotonic dystrophy. Acta Neurol Scand. 2016;134:346-51.

16. Regnault A, Willgoss $\mathrm{T}$, Barbic S. Towards the use of mixed methods inquiry as best practice in health outcomes research. J Patient-Reported Outcomes. 2018;2:19.

17. Morel T, Cano SJ. Measuring what matters to rare disease patients-reflections on the work by the IRDiRC taskforce on patient-centered outcome measures. Orphanet J Rare Dis. 2017; 12:171

18. U.S. Food and Drug Administration. Patient-focused drug development: methods to identify what is important to patients. Rockville; 2019.

19. Petrovski S, Wang Q, Heinzen EL, Allen AS, Goldstein DB. Genic intolerance to functional variation and the interpretation of personal genomes. Williams SM, editor. PLoS Genet. 2013;9:e1003709.

20. Todd JJ, Lawal TA, Witherspoon JW, Chrismer IC, Razaqyar MS, Punjabi M, et al. Randomized controlled trial of $N$-acetylcysteine therapy for RYR1-related myopathies. Neurology. 2020. https:// doi.org/10.1212/WNL.0000000000008872.
21. Amburgey K, McNamara N, Bennett LR, McCormick ME, Acsadi G, Dowling JJ. Prevalence of congenital myopathies in a representative pediatric United States population. Ann Neurol. 2011;70:662-5.

22. Colombo I, Scoto M, Manzur AY, Robb SA, Maggi L, Gowda V, et al. Congenital myopathies: natural history of a large pediatric cohort. Neurology. 2015;84:28-35.

23. Todd JJ, Razaqyar MS, Witherspoon JW, Lawal TA, Mankodi A, Chrismer IC, et al. Novel variants in individuals with RYR1related congenital myopathies: genetic, laboratory, and clinical findings. Front Neurol. 2018;9.

24. Dlamini N, Voermans NC, Lillis S, Stewart K, Kamsteeg E-J, Drost $\mathrm{G}$, et al. Mutations in RYR1 are a common cause of exertional myalgia and rhabdomyolysis. Neuromuscul Disord. 2013;23:540-8.

25. van Ruitenbeek E, Custers JAE, Verhaak C, Snoeck M, Erasmus $\mathrm{CE}$, Kamsteeg EJ, et al. Functional impairments, fatigue and quality of life in RYR1-related myopathies: a questionnaire study. Neuromuscul Disord. 2019;29:30-8.

26. Klein A, Jungbluth $\mathrm{H}$, Clement E, Lillis S, Abbs S, Munot P, et al Muscle magnetic resonance imaging in congenital myopathies due to ryanodine receptor type 1 gene mutations. Arch Neurol. 2011;68:1171-9.

27. Matthews E, Neuwirth C, Jaffer F, Scalco RS, Fialho D, Parton $\mathrm{M}$, et al. Atypical periodic paralysis and myalgia. Neurology. 2018;90:e412-e418418.

28. Lawal TA, Todd JJ, Meilleur KG. Ryanodine receptor 1-related myopathies: Diagnostic and therapeutic approaches. Neurotherapeutics. 2018;15:885-99.

29. Zhou H, Jungbluth H, Sewry CA, Feng L, Bertini E, Bushby K, et al. Molecular mechanisms and phenotypic variation in RYR1related congenital myopathies. Brain. 2007;130:2024-36.

30. Dowling JJ, Arbogast S, Hur J, Nelson DD, McEvoy A, Waugh $\mathrm{T}$, et al. Oxidative stress and successful antioxidant treatment in models of RYR1-related myopathy. Brain. 2012;135:1115-27.

31. Durham WJ, Aracena-Parks P, Long C, Rossi AE, Goonasekera SA, Boncompagni S, et al. RyR1 S-nitrosylation underlies environmental heat stroke and sudden death in Y522S RyR1 knockin mice. Cell. 2008;133:53-655.

32. Lee CS, Hanna AD, Wang H, Dagnino-Acosta A, Joshi AD, Knoblauch $\mathrm{M}$, et al. A chemical chaperone improves muscle function in mice with a RyR1 mutation. Nat Commun (Nature Publishing Group). 2017;8:14659.

33. Creswell JW, Plano Clark VL. Designing and conducting mixed methods research. Thousand Oaks: Sage Publications; 2007.

34. Creswell JW. Research design. Qualitative, quantitative and mixed methods approaches. 4th ed. London: Sage Publications; 2014.

35. Geirdal AØ, Lund-Petersen I, Heiberg A. Understanding the experience of myotonic dystrophy. Mixed method study. J Genet Couns. 2015;24:169-78.

36. Johnson NE, Luebbe E, Eastwood E, Chin N, Moxley RT, Heatwole CR. The impact of congenital and childhood myotonic dystrophy on quality of life: a qualitative study of associated symptoms. J Child Neurol. 2014;29:983-6.

37. Vorster N, Evans K, Murphy N, Kava M, Cairns A, Clarke D, et al. Powered standing wheelchairs promote independence, health and community involvement in adolescents with Duchenne muscular dystrophy. Neuromuscul Disord. 2019;29:221-30.

38. Sherman DW, McGuire DB, Free D, Cheon JY. A pilot study of the experience of family caregivers of patients with advanced pancreatic cancer using a mixed methods approach. J Pain Symptom Manag. 2014;48(385-399):e2.

39. Wilkinson S. Women with breast cancer talking causes: comparing content, biographical and discursive analyses. Fem Psychol. 2000;10:431-60. 
40. Palinkas LA, Horwitz SM, Green CA, Wisdom JP, Duan N, Hoagwood K. Purposeful sampling for qualitative data collection and analysis in mixed method implementation research. Adm Policy Ment Heal Ment Heal Serv Res. 2015;42:533-44.

41. Braun V, Clarke V. Using thematic analysis in psychology. Brooks J, King N, editors. Qual Res Psychol. London: Macmillan Education UK; 2006;3:77-101.

42. Flick U. An introduction to qualitative research. 5 th ed. Thousand Oaks: Sage Publications; 2014.

43. Saunders B, Sim J, Kingstone T, Baker S, Waterfield J, Bartlam $\mathrm{B}$, et al. Saturation in qualitative research: exploring its conceptualization and operationalization. Qual Quant. 2018;52:1893-907.

44. LaFaver K, Miyasaki JM, Keran CM, Rheaume C, Gulya L, Levin $\mathrm{KH}$, et al. Age and sex differences in burnout, career satisfaction, and well-being in US neurologists. Neurology. 2018;91:e1928-e19411941.

45. Miyasaki JM, Rheaume C, Gulya L, Ellenstein A, Schwarz HB, Vidic TR, et al. Qualitative study of burnout, career satisfaction, and well-being among US neurologists in 2016. Neurology. 2017;89:1730-8.

46. Wei Y, Speechley K, Campbell C. Health-related quality of life in children with Duchenne muscular dystrophy: a review. J Neuromuscul Dis. 2015;2:313-24.

47. Winter Y, Schepelmann K, Spottke AE, Claus D, Grothe C, Schröder R, et al. Health-related quality of life in ALS, myasthenia gravis and facioscapulohumeral muscular dystrophy. J Neurol. 2010;257:1473-81.

48. Frisaldi E, Shaibani A, Vollert J, Ferrero B, Carrino R, Ibraheem $\mathrm{HD}$, et al. The placebo response in myasthenia gravis assessed by quantitative myasthenia gravis score: a meta-analysis. Muscle Nerve (internet). 2019;59:671-8. https://onlinelibrary.wiley.com/ doi/abs/10.1002/mus.26469.

49. Shaibani A, Frisaldi E, Benedetti F. Placebo response in pain, fatigue, and performance: possible implications for neuromuscular disorders. Muscle Nerve. 2017;56:358-67.
50. Dowling JJ, Lillis S, Amburgey K, Zhou H, Al-Sarraj S, Buk SJA, et al. King-Denborough syndrome with and without mutations in the skeletal muscle ryanodine receptor (RYR1) gene. Neuromuscul Disord. 2011;21:420-7.

51. Witting N, Werlauff U, Duno M, Vissing J. Phenotypes, genotypes, and prevalence of congenital myopathies older than 5 years in Denmark. Neurol Genet. 2017;3:e140.

52. Jungbluth H, Müller CR, Halliger-Keller B, Brockington M, Brown SC, Feng L, et al. Autosomal recessive inheritance of RYR1 mutations in a congenital myopathy with cores. Neurology. 2002;59:284-7.

53. Jungbluth $\mathrm{H}$, Zhou H, Hartley L, Halliger-Keller B, Messina S, Longman C, et al. Minicore myopathy with ophthalmoplegia caused by mutations in the ryanodine receptor type 1 gene. Neurology. 2005;65:1930-5.

54. Scacheri PC, Hoffman EP, Fratkin JD, Semino-Mora C, Senchak A, Davis MR, et al. A novel ryanodine receptor gene mutation causing both cores and rods in congenital myopathy. Neurology. 2000;55:1689-96.

55. Kelly GS. Clinical applications of $N$-acetylcysteine. Altern Med Rev. 1998;3:114-27.

56. Capacchione JF, Sambuughin N, Bina S, Mulligan LP, Lawson TD, Muldoon SM. Exertional rhabdomyolysis and malignant hyperthermia in a patient with ryanodine receptor type 1 gene, L-type Calcium Channel A-1 Subunit Gene, And Calsequestrin-1 Gene Polymorphisms. Anesthesiology. 2010;112:239-44.

57. Davis M, Brown R, Dickson A, Horton H, James D, Laing N, et al. Malignant hyperthermia associated with exercise-induced rhabdomyolysis or congenital abnormalities and a novel RYR1 mutation in New Zealand and Australian pedigrees. Br J Anaesth. 2002;88:508-15.

58. Bingel U, Wanigasekera V, Wiech K, Ni Mhuircheartaigh R, Lee $\mathrm{MC}$, Ploner M, et al. The effect of treatment expectation on drug efficacy: Imaging the analgesic benefit of the opioid Remifentanil. Sci Transl Med. 2011;3:70ra14. 